\title{
Adrenalectomy for Primary Aldosteronism: Significant Variability in Work-Up Strategies and Low Guideline Adherence in Worldwide Daily Clinical Practice
}

\author{
Wessel M. C. M. Vorselaars ${ }^{1} \cdot$ Dirk-Jan van Beek $^{1} \cdot$ Diederik P. D. Suurd $^{1}$ • \\ Emily Postma $^{1}$ - Wilko Spiering ${ }^{2}$ Inne H. M. Borel Rinkes ${ }^{1} \cdot$ Gerlof D. Valk $^{3}$ • \\ Menno R. Vriens ${ }^{1} \cdot$ International CONNsortium*
}

Published online: 5 February 2020

(C) The Author(s) 2020

\begin{abstract}
Background Various diagnostic tests are available to establish the primary aldosteronism (PA) diagnosis and to determine the disease laterality. Combined with the controversies in the literature, unawareness of guidelines and technical demands and high costs of some of these diagnostics, this could lead to significant differences in work-up strategies worldwide. Therefore, we investigated the work-up before surgery for PA in daily clinical practice within a multicenter study.

Methods Patients who underwent unilateral adrenalectomy for PA within 16 centers in Europe, Canada, Australia and the USA between 2010 and 2016 were included. We did not exclude patients based on the performed diagnostic tests during work-up to make our data representative for current clinical practice. Adherence to the Endocrine Society Guideline and variables associated with not performing adrenal venous sampling (AVS) were analyzed.

Results In total, 435 patients were eligible. An aldosterone-to-renin ratio, confirmatory test, computed tomography (CT), magnetic resonance imaging and AVS were performed in $82.9 \%, 32.9 \%, 86.9 \%, 17.0 \%$ and $65.3 \%$ of patients, respectively. A complete work-up, as recommended by the guideline, was performed in $13.1 \%$ of patients. Bilateral disease or normal adrenal anatomy on CT (OR 16.19; CI 3.50-74.99), smaller tumor size on CT (OR 0.06; CI 0.04-0.08) and presence of hypokalemia (OR 2.00; CI 1.19-3.32) were independently associated with performing AVS.

Conclusions This study is the first to examine the daily clinical practice work-up of PA within a worldwide cohort of surgical patients. The results demonstrate significant variability in work-up strategies and low adherence to The Endocrine Society guideline.
\end{abstract}

Menno R. Vriens

mvriens@umcutrecht.nl

1 Department of Surgical Oncology and Endocrine Surgery, University Medical Center Utrecht, Room G04.228, Heidelberglaan 100, 3584 CX Utrecht, The Netherlands

2 Department of Vascular Medicine, University Medical Center Utrecht, Utrecht, The Netherlands

3 Department of Endocrine Oncology, University Medical Center Utrecht, Utrecht, The Netherlands

\section{Introduction}

Primary aldosteronism (PA) is the most common surgically treatable cause of secondary hypertension with an estimated prevalence of 5-20\% within the hypertensive population [1-7]. In the vast majority of cases, PA is either caused by bilateral adrenal hyperplasia or by a unilateral aldosterone-producing adenoma (APA). While bilateral hyperplasia is generally treated with a mineralocorticoid receptor agonist, adrenalectomy is the preferred treatment for patients with APA $[8,9]$. 
In 2008, The Endocrine Society published a clinical practice guideline on PA with the goal of improving screening, work-up and treatment of PA worldwide [8]. The guideline recommended the use of the aldosterone-torenin ratio (ARR) to detect cases of PA among hypertensive patients. Due to the risk of false-positive ARRs, case confirmation with a confirmatory test was recommended in all patients with a positive ARR. Computed tomography (CT) was recommended to exclude adrenocortical carcinoma and in case surgery for PA was indicated, and adrenal venous sampling (AVS) was recommended in all patients to distinguish APA from bilateral hyperplasia [8]. In 2016, an update of the Endocrine Society Guideline was published [9]. This revised guideline suggested that a specific subgroup of patients potentially do not have to undergo confirmatory testing or AVS. However, these recommendations were based on a relatively low level of evidence [9].

Within the work-up to adrenalectomy in patients with PA, however, a large variety of diagnostic tests and imaging modalities are available to establish the PA diagnosis and to determine laterality of disease. This is reflected in the numerous controversies in the literature and between experts in the field regarding the different preoperative work-up strategies. Currently, the most important topic of discussion is whether all patients should undergo confirmatory testing and AVS [10-12]. Moreover, these diagnostics could be considered expensive, laborious or technically demanding. Based on the above, we hypothesized that clinicians might deviate from the Endocrine Society guideline within current daily clinical practice.

In the past, complete cure of hypertension after the operation was estimated in approximately $50 \%$ of patients $[13,14]$. However, recently the Primary Aldosteronism Surgery Outcome (PASO) study group and our own study group showed the less optimistic results by presenting a 27-37\% cure rate within large, international and well-executed studies $[12,15,16]$. This stresses the need to evaluate current practice with the goal to improve the benefits of surgery. Since the present literature lacks data on how the work-up to surgery is performed in daily practice, we set out to evaluate and describe the performed work-up within a worldwide cohort of patients who underwent unilateral adrenalectomy for PA between 2010 and 2016.

\section{Methods}

\section{Study population}

We performed an international retrospective cohort study across 16 referral medical centers in The USA, Europe,
Canada and Australia. The study cohort was established by the International CONNsortium study group and the derivation of the cohort and the blood pressure-related outcomes has been described in previous publications $[15,16]$. In brief, all consecutive patients who underwent unilateral total adrenalectomy for APA between 2010 and 2016 were included retrospectively. We did not include or exclude patients based on the performed work-up strategy and, therefore, the diagnosis of PA and indication for surgery were based on the treating physicians' assessment and discretion. In the majority of cases, biochemical evidence of PA was based on the ARR (or in some cases single aldosterone) measurements. Confirmatory testing was performed according to the physicians' preference and/or availability of this test within each medical center. Unilateral disease was diagnosed based on CT and/or magnetic resonance imaging (MRI) and/or AVS according to the preference of the physician and/or availability of these modalities within each medical center. Since the cohort was initiated for a different study aim (i.e., to describe the reduction in blood pressure and antihypertensive medications after adrenalectomy), patients with missing preoperative or follow-up data regarding systolic blood pressure (SBP), diastolic blood pressure (DBP) or corresponding number of antihypertensive medications were not included in the cohort [15]. Data collection was performed separately within each center with the use of a standardized data-entry manual. Patient demographics, disease characteristics, laboratory data (e.g., measurements of ARR and confirmatory testing), results of CT/MRI/AVS, operative characteristics, pathology diagnosis and timing of follow-up were collected. Institutional review board approval was obtained in all participating centers.

\section{Outcomes}

The primary aim of this study was to evaluate the adherence to The Endocrine Society guideline for the work-up of patients treated for PA [8]. As our cohort consisted of who had an adrenalectomy before (or around) publication of the most recent 2016 version of the guideline, we chose to primarily compare our results to the guideline which was published in 2008 [8]. Within the new 2016 guideline, only two recommendations regarding work-up were introduced:

I. In case of hypokalemia, plasma renin levels below detection levels and aldosterone above $>20 \mathrm{ng} / \mathrm{dL}$ $(550 \mathrm{pmol} / \mathrm{L})$, no confirmatory testing may be needed;

II. In case of age $<35$ years old, hypokalemia, marked aldosterone excess and unilateral cortical adenoma on CT, no AVS may be needed [9].

We additionally aimed to evaluate the potential influence of these new recommendations on clinical practice by 
examining the proportions of patients fulfilling/meeting these criteria within our cohort.

The secondary aim of this study was to identify potential disease or patient characteristics which encouraged clinicians to distinguish APA from bilateral hyperplasia and to determine laterality of disease based on CT alone without performing AVS.

\section{Definitions}

Due to the different assays and reference values within the participating centers, we were not able to analyze absolute values of biochemical measurements. To compare laboratory data between the centers, measurements were classified as elevated or suppressed when they were above the upper or below the lower limit of the center's local reference ranges, respectively. Marked aldosterone excess was defined as an elevated aldosterone level, and hypokalemia was defined as either a potassium level below the local reference range or the use of potassium supplementation. When the results of biochemical measurements (e.g., ARR or confirmatory test) were not known within the operating centers, the measurement was reported as not performed. When these measurements were performed in other medical centers before referral, the results were reported within the database. Complete cure of hypertension was defined according to the PASO consensus criteria [12].

\section{Statistical analysis}

Normally and not normally distributed continuous data are shown as mean ( \pm standard deviation) and median (range). To compare continuous variables between groups, the Mann-Whitney $U$ Test was used for not normally distributed data and independent samples t tests for normally distributed data. The Chi-Square test and Fisher's exact test were used to analyze group differences for categorical variables. To analyze potential variables associated with not making use of AVS, we performed multivariable logistic regression with backward stepwise selection including variables with $p<0.25$ in univariable analysis. Only patients who underwent CT were included in this analysis. Multiple potential prognostic variables had missing values. These variables were imputed using multiple imputation generating 20 imputed datasets [17]. Outcomes were not imputed. Pooled odds ratios with $95 \%$ confidence intervals were obtained from multivariable logistic regression. All tests were two-sided and p-values $<$ 0.05 were considered significant. Statistical analysis was performed using SPSS version 23.0 (Chicago, Illinois, USA), and figures were constructed using Graphpad Prism version 7.02 (GraphPad Software Inc, California, USA) and Draw.io version 10.5-1 (JGraph Ltd, Northamptonshire, UK).

\section{Results}

Four hundred and thirty-five (85\%) patients were eligible for analysis [15]. Baseline characteristics of these patients are presented in Table 1. Most patients were men (57.2\%). The mean age and mean BMI were $50.7 \pm 11.4$ years and $29.7 \pm 6.0 \mathrm{~kg} / \mathrm{m}^{2}$, respectively. Hypokalemia was present in $73.9 \%$ of patients, and most patients had grade 1 hypertension (41.4\%). Preoperative work-up data of these patients are presented in Table 2. In $82.9 \%$ of patients, a complete measurement of the ARR was performed and in 94.5\% of these patients, the ARR was elevated indicating PA. A confirmatory test was performed in $32.9 \%$ of all patients, indicating PA in $89.5 \%$. CT, MRI and AVS were performed in $86.9 \%, 17.0 \%$ and $65.3 \%$ of the cohort, respectively. Almost half of the patients $(49.9 \%)$ underwent both CT and AVS for subtype testing. CT only, MRI

Table 1 Baseline characteristics of 435 patients

\begin{tabular}{ll}
\hline Variable & $\begin{array}{l}\text { Number }(\%) \text { or } \\
\text { mean } \pm \text { SD }\end{array}$ \\
\hline Age at surgery (years) & $50.7 \pm 11.4$ \\
Female & $186(42.8 \%)$ \\
Duration of hypertension (years) & $9(0-42)$ \\
$\quad(n=366) *$ & \\
Body mass index $\left(\mathrm{kg} / \mathrm{m}^{2}\right)(n=402)$ & $29.7 \pm 6.0$ \\
Number of antihypertensive medications & $3(0-8)$ \\
Defined daily dose $(n=405)^{*}$ & $3.7(0.0-25.3)$ \\
Hypokalemia $(n=429)$ & $317(73.9 \%)$ \\
Preoperative mean SBP $(\mathrm{mmHg})$ & $150 \pm 20$ \\
Preoperative mean DBP $(\mathrm{mmHg})$ & $90 \pm 13$ \\
JNC/ESH hypertension grade based on blood pressure with \\
$\quad$ medication & $111(25.5 \%)$ \\
Grade 0 & $180(41.4 \%)$ \\
Grade 1 & $105(24.1 \%)$ \\
Grade 2 & $39(9.0 \%)$ \\
Grade 3 & \\
Surgical procedure & $171(39.3 \%)$ \\
EPRA & $65(14.9 \%)$ \\
ELRA & $198(45.5 \%)$ \\
LTA & $1(0.2 \%)$ \\
Open &
\end{tabular}

*Values not normally distributed given as medians (range)

$J N C=$ Joint National Commission, $E S H=$ European Society of Hypertension, $S B P=$ systolic blood pressure, $D B P=$ diastolic blood pressure, $E P R A=$ endoscopic posterior retroperitoneal adrenalectomy, $E L R A=$ endoscopic lateral retroperitoneal adrenalectomy, $L T A=$ laparoscopic transabdominal adrenalectomy 
Table 2 Preoperative work-up

\begin{tabular}{|c|c|}
\hline Variable & Number $(\%)$ \\
\hline Measurement of aldosterone performed & $408(93.8 \%)$ \\
\hline Aldosterone elevated & $225(55.1 \%)$ \\
\hline Measurement of renin performed & $370(85.1 \%)$ \\
\hline Renin suppressed & $245(66.2 \%)$ \\
\hline Measurement of ARR performed & $361(82.9 \%)$ \\
\hline ARR elevated & $341(94.5 \%)$ \\
\hline Confirmatory test performed & $143(32.9 \%)$ \\
\hline Oral salt loading & $18(12.6 \%)$ \\
\hline Saline infusion test & $118(82.5 \%)$ \\
\hline Fludrocortisone suppression test & $3(2.1 \%)$ \\
\hline Captopril challenge & $1(0.7 \%)$ \\
\hline Fludrocortisone dexamethasone suppression test & $1(0.7 \%)$ \\
\hline $\begin{array}{l}\text { Post-low dose dexamethasone suppression-saline } \\
\text { infusion test }\end{array}$ & $1(0.7 \%)$ \\
\hline \multicolumn{2}{|l|}{ Confirmatory test indicating PA } \\
\hline Yes & $128(89.5 \%)$ \\
\hline No & $13(9.1 \%)$ \\
\hline Missing data & $2(1.4 \%)$ \\
\hline CT performed & $378(86.9 \%)$ \\
\hline Unilateral disease & $325(86.0 \%)$ \\
\hline Bilateral disease & $28(7.4 \%)$ \\
\hline Normal adrenal anatomy & $21(5.6 \%)$ \\
\hline Missing data & $4(1.1 \%)$ \\
\hline MRI performed & $72(17 \%)$ \\
\hline Unilateral disease & $63(87.5 \%)$ \\
\hline Bilateral disease & $3(4.2 \%)$ \\
\hline Normal adrenal anatomy & $5(6.9 \%)$ \\
\hline Missing data & $1(1.9 \%)$ \\
\hline AVS performed & $284(65.3 \%)$ \\
\hline Unilateral disease & $263(92.6 \%)$ \\
\hline Bilateral disease & $7(2.5 \%)$ \\
\hline No lateralization & $7(2.5 \%)$ \\
\hline Failure of procedure & $6(2.1 \%)$ \\
\hline Missing data & $1(0.4 \%)$ \\
\hline
\end{tabular}

$A R R=$ aldosterone-to-renin ratio, $P A=$ primary aldosteronism, $C T=$ computerized tomography, $M R I=$ magnetic resonance imaging, $A V S=$ adrenal venous sampling

only and AVS only were used in $28.5 \%, 5.1 \%$ and $3.7 \%$ of patients, respectively. Furthermore, CT combined with MRI was used in $3.9 \%$ and MRI combined with AVS in $4.4 \%$ of patients. All three modalities were used in $4.6 \%$ of patients (Fig. 1). As indicated in Fig. 2, large variability in work-up strategies was observed between the different medical centers. Depending on the medical center, the use of a confirmatory test, CT and AVS ranged from 0.0 to $94.6 \%, 66.7$ to $100.0 \%$ and 9.1 to $100.0 \%$ of patients, respectively. All centers used AVS in some cases, and only

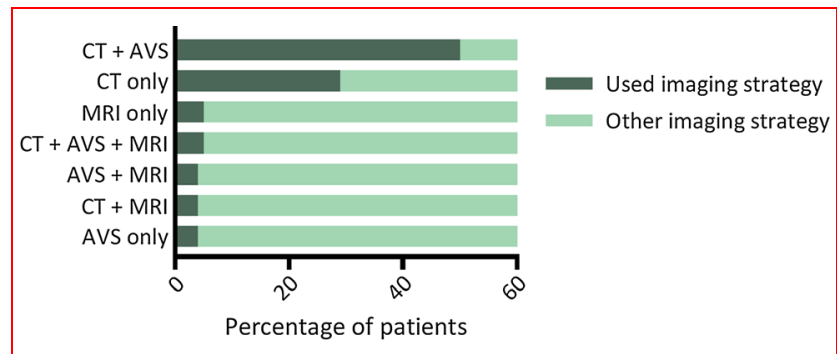

Fig. 1 Imaging modalities used for subtype testing. $C T=$ computerized tomography, $M R I=$ magnetic resonance imaging; $A V S=$ adrenal venous sampling

one center performed AVS in all cases. Furthermore, MRI was used in all medical centers except one.

\section{Adherence to the 2008 endocrine society guideline}

Out of the 435 patients who underwent surgery for PA, screening was performed by a complete ARR in 361 patients (83.0\%) and in 341 patients (78.4\%), this ARR was elevated suggesting PA (Fig. 3). Of the patients without a preoperative ARR, a preoperative aldosterone measurement was performed in $63.5 \%$ of patients showing elevated aldosterone levels in $72.3 \%$ of these patients. A confirmatory test was performed in 114 of the 341 patients with an elevated ARR (33.4\%), and in 102 patients (29.9\%) the test indicated PA. Ninety-one of these 102 patients (89.2\%) underwent CT, and in 11 patients $(10.8 \%)$ no CT was performed. These 11 patients underwent MRI and/or AVS. Sixty out of 91 patients $(65.9 \%)$ also underwent AVS, and in $57(62.6 \%)$ patients the AVS indicated unilateral disease. When combining these results, 57 out of the $435(13.1 \%)$ patients who had surgery within this cohort underwent the complete work-up as recommended by the 2008 Endocrine Society Guideline [8]. All other patients did not undergo all recommended diagnostic modalities or, for instance, had an ARR or confirmatory test not compatible with PA (Fig. 3). Complete cure of hypertension after the operation was comparable between the $13.1 \%$ of patients with the complete work-up and all other patients, $30 \%$ and $27 \%$, respectively.

Fig. 2 Large heterogeneity in the use of confirmatory testing, MRI, CT and AVS. $C T=$ computerized tomography, $M R I=$ magnetic resonance imaging, $A V S=$ adrenal venous sampling. Legend: This figure shows the use of different diagnostic modalities in the work-up of primary aldosteronism specified by continent and medical center. As presented, a large variability in work-up strategies was used in daily clinical practice and, in contrast to the guideline, confirmatory testing and AVS were not regularly performed 

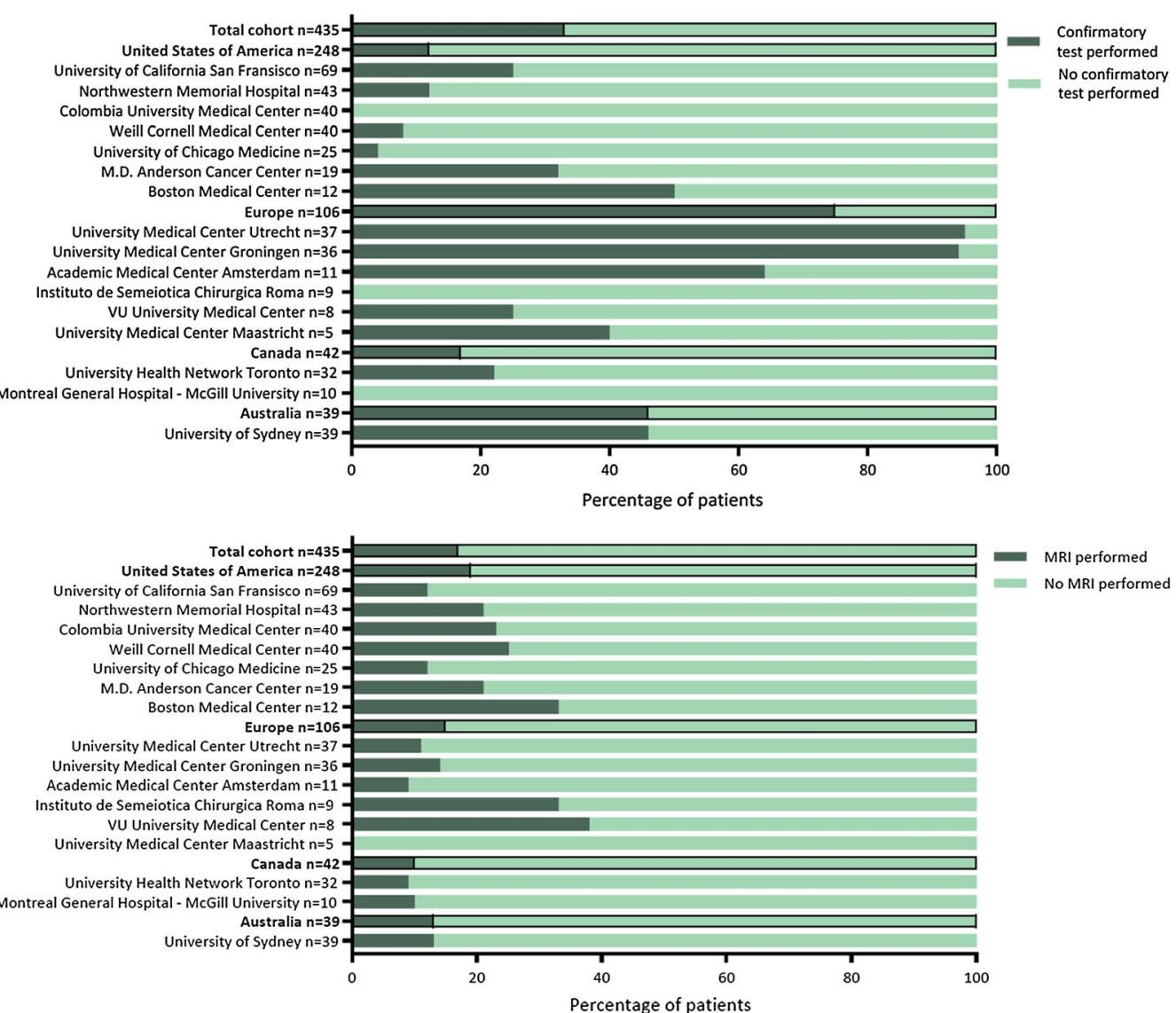

MRI performed

No MRI performed

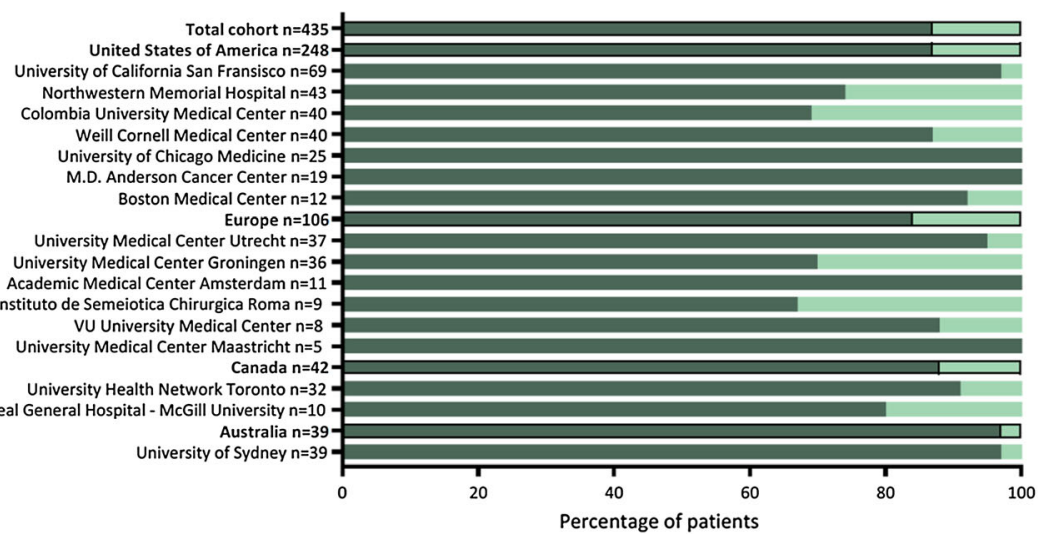

CT performed

No CT performed

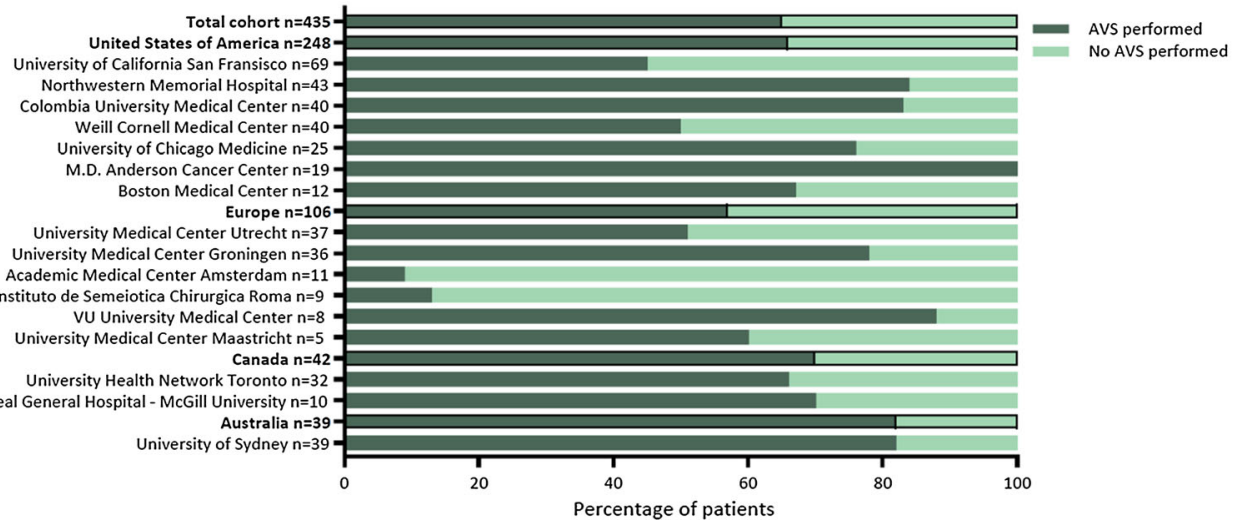


Endocrine Society Guideline 2008

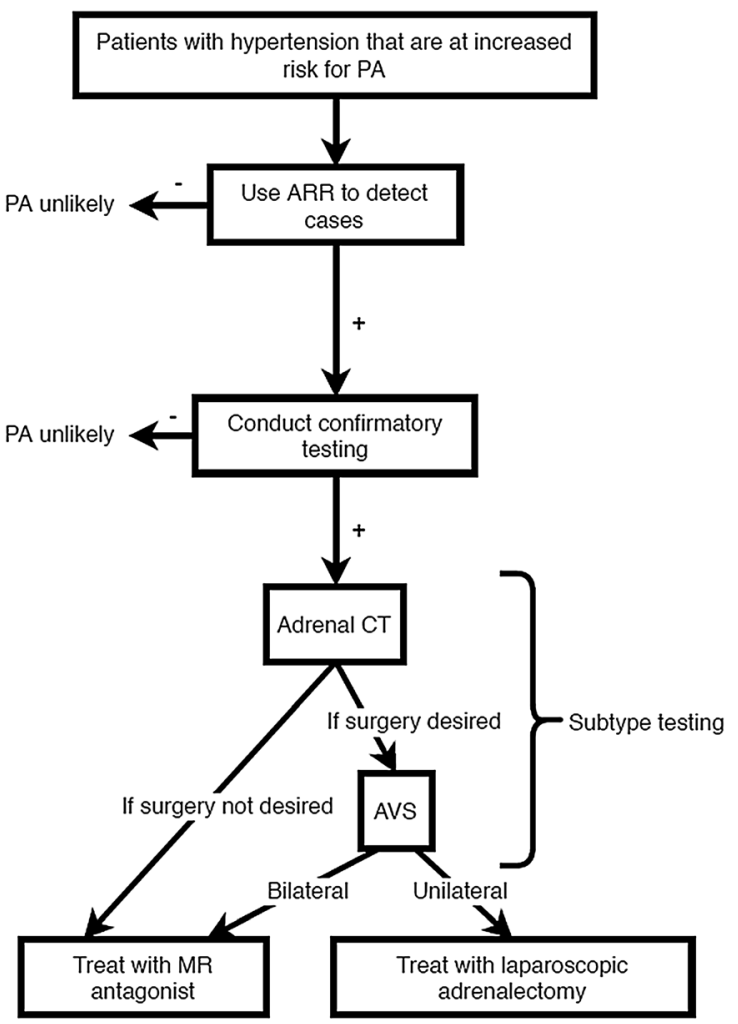

Clinical Practice

Patients treated with unilateral laparoscopic adrenalectomy $n=435(100 \%)$

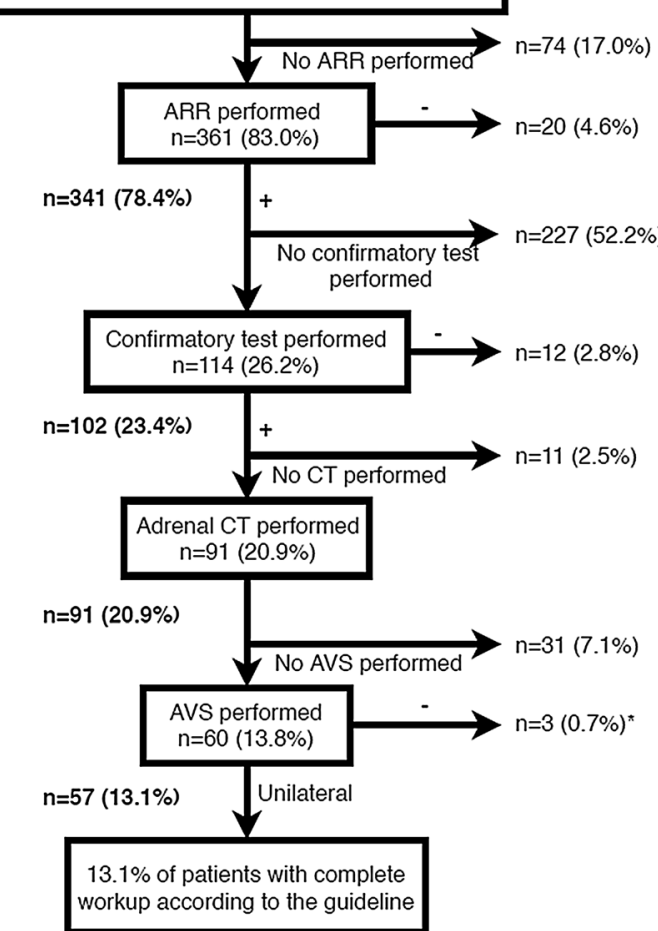

Fig. 3 Work-up in current clinical practice in contrast to the Endocrine Society Guideline 2008. *2 patients had failure of AVS procedure and in 1 patient AVS showed no lateralization. $P A=$ primary aldosteronism, $A R R=$ aldosterone-to-renin ratio, $C T=$ computerized tomography, $M R I=$ magnetic resonance imaging, $A V S=$ adrenal venous sampling. Legend: This figure describes the work-up to surgery for primary aldosteronism in daily clinical practice as it was performed within this study cohort. The performed work-up was compared to the 2008 Endocrine Society Guideline as this was accurate during the inclusion period of the study. The figure shows low guideline adherence since $13.1 \%$ underwent the complete work-up according to the guideline. Patients were excluded when: (1) they did not undergo one of the recommended diagnostic test, (2) the results of the aldosterone-to-renin ratio and confirmatory test did not correlate with primary aldosteronism, (3) the results of adrenal venous sampling did not indicate unilateral aldosterone hypersecretion (all indicated by the horizontal arrows to the right)

\section{Evaluation of the 2016 endocrine society guideline}

Within the complete cohort, 177 patients $(40.7 \%)$ preoperatively had known elevated aldosterone, suppressed renin and spontaneous hypokalemia omitting the need for confirmatory testing according to the revised 2016 guideline. Only 49 (18.9\%) of the 258 patients that did not meet these criteria underwent confirmatory testing. Among the 242 patients in whom a preoperative CT was performed, only $30(12.4 \%)$ patients were younger than 35 years of age and only $14(5.8 \%)$ patients also had an elevated aldosterone, spontaneous hypokalemia and a unilateral nodule on CT. According to the 2016 guideline, these $5.8 \%$ of patients did not have to undergo AVS [9].

\section{Variables associated with performing AVS}

Univariable analysis showed that AVS was more frequently performed in case of older age, male gender, longer duration of hypertension, presence of hypokalemia, CT indicating bilateral disease or normal adrenal anatomy and a smaller tumor on CT (Table 3). After multivariable regression analysis, bilateral disease or normal adrenal anatomy on CT (OR 16.19; CI 3.50-74.99) $(p<0.001)$, smaller tumor size on CT (mm) (OR 0.06; CI 0.04-0.08) $(p<0.001)$ and hypokalemia (OR 2.00; CI 1.19-3.32) $(p=0.008)$ remained independently associated with performing AVS. 
Table 3 Variables associated with performing AVS for subtype testing

\begin{tabular}{|c|c|c|c|c|c|c|c|}
\hline & \multicolumn{5}{|c|}{ Univariable analysis } & \multicolumn{2}{|c|}{$\begin{array}{l}\text { Multivariable regression } \\
\text { analysis with backward } \\
\text { selection** }\end{array}$} \\
\hline & $\begin{array}{l}\% \\
\text { Missing }\end{array}$ & $\begin{array}{l}\text { CT }+ \text { AVS } \\
(n=242)\end{array}$ & $\begin{array}{l}\text { CT only } \\
(n=136)\end{array}$ & OR $(95 \% \mathrm{CI})$ & $p$ value & OR $(95 \% \mathrm{CI})$ & $p$ value \\
\hline Age (years) & 0.0 & $51.6(11.0)$ & $48.9(11.1)$ & $1.02(1.00-1.04)$ & 0.026 & - & NS \\
\hline Gender & 0.0 & & & & & & \\
\hline Male & & $153(71.8 \%)$ & $60(28.2 \%)$ & $2.18(1.42-3.34)$ & $<0.001$ & - & NS \\
\hline Female & & $89(53.9 \%)$ & $76(46.1 \%)$ & 1 (ref) & & & \\
\hline Body mass index $\left(\mathrm{kg} / \mathrm{m}^{2}\right)$ & 7.7 & $30.0(6.2)$ & $29.0(6.2)$ & $1.03(0.99-1.06)$ & 0.153 & - & \\
\hline $\begin{array}{l}\text { Duration of hypertension } \\
\text { (years)* }\end{array}$ & 14.8 & $10(0-40)$ & $7(0-38)$ & $1.04(1.01-1.08)$ & 0.016 & - & \\
\hline $\begin{array}{l}\text { Systolic blood pressure } \\
\quad(\mathrm{mmHg})\end{array}$ & 0.0 & $150 \pm 19$ & $149 \pm 18$ & $1.00(0.99-1.01)$ & 0.635 & NA & NA \\
\hline $\begin{array}{l}\text { Diastolic blood pressure } \\
\quad(\mathrm{mmHg})\end{array}$ & 0.0 & $90 \pm 12$ & $88 \pm 11$ & $1.02(1.00-1.04)$ & 0.067 & - & NS \\
\hline Number of antihypertensives* & 0.0 & $3(0-8)$ & $3(0-7)$ & $1.03(0.88-1.21)$ & 0.846 & NA & NA \\
\hline Defined daily dose* & 6.6 & $3.7(0.0-22.3)$ & $3.2(0.0-25.3)$ & $1.03(0.97-1.10)$ & 0.332 & NA & NA \\
\hline ARR indicating PA & 16.9 & & & & & & \\
\hline Yes & & $187(63.6 \%)$ & $107(36.4 \%)$ & $1.75(0.71-4.33)$ & 0.223 & - & NS \\
\hline No & & $10(50.0 \%)$ & $10(50.0 \%)$ & 1 (ref) & & & \\
\hline Elevated aldosterone & 6.9 & & & & & & \\
\hline Yes & & $129(66.2 \%)$ & $66(33.8 \%)$ & $1.24(0.80-$ & 0.331 & NA & NA \\
\hline No & & $96(61.1 \%)$ & $61(38.9 \%)$ & 1.92) 1 (ref) & & & \\
\hline Suppressed renin & 8.5 & & & & & & \\
\hline Yes & & $137(64.6 \%)$ & $75(35.4 \%)$ & $1.41(0.88-2.26)$ & 0.156 & - & NS \\
\hline No & & $61(56.5 \%)$ & $47(43.5 \%)$ & 1 (ref) & & & \\
\hline Hypokalemia & 1.3 & & & & & & \\
\hline Yes & & $188(67.6 \%)$ & $90(32.4 \%)$ & $1.88(1.17-3.02)$ & 0.009 & $2.00(1.19-3.32)$ & 0.008 \\
\hline No & & $50(52.6 \%)$ & $45(47.4 \%)$ & 1 (ref) & & 1 (ref) & \\
\hline CT outcome & 1.1 & & & & & & \\
\hline Unilateral nodule & & $192(59.1 \%)$ & $133(40.9 \%)$ & 1 (ref) & $<0.001$ & 1 (ref) & $<0.001$ \\
\hline No unilateral nodule & & $47(96.1 \%)$ & $2(3.9 \%)$ & $\begin{array}{l}16.28 \\
\quad(3.89-68.18)\end{array}$ & & $\begin{array}{l}16.19 \\
\quad(3.50-74.99)\end{array}$ & \\
\hline Bilateral nodule & & $26(92.9 \%)$ & $2(7.1 \%)$ & & & & \\
\hline Normal anatomy & & $21(100 \%)$ & $0(0 \%)$ & & & & \\
\hline CT tumor size $(\mathrm{mm})^{*}$ & $6.8 \%$ & $14(0-29)$ & $16(6-95)$ & $0.05(0.04-0.07)$ & $<0.001$ & $0.06(0.04-0.08)$ & $<0.001$ \\
\hline
\end{tabular}

Bold values indicate statistical significance $(p<0.05)$

*Values not normally distributed given as medians (range)

**Variables with a $p$ value $<0.25$ after univariable analysis were used for multivariable regression analysis with backward selection $A R R=$ aldosterone-to-renin ratio, $P A=$ primary aldosteronism, $C T=$ computerized tomography, $A V S=$ adrenal venous sampling, $O R=$ odds ratio, $C I=$ confidence interval, $r e f=$ reference variable, $N S=$ not significant, $N A=$ not applicable

\section{Discussion}

This study evaluated the work-up to adrenalectomy for PA within current daily practice in an international retrospective cohort of surgical patients. The results displayed a large variability in work-up strategies between the participating centers worldwide. During the inclusion period of this study, the 2008 Endocrine Society Guideline was applicable [8]. Only $13.1 \%$ of the operated patients underwent a complete work-up as was recommended by this guideline. Although almost all patients underwent CT (or MRI), confirmatory testing and AVS were performed in 
only one-third and two-third of the operated patients, respectively. The results also showed a significant variability in work-up within the majority of centers. Moreover, this variability in work-up was also shown between countries, between the centers within these countries and within the individual centers. Therefore, this study illustrates that clinicians most likely chose a particular work-up strategy, such as the selective use of AVS, based on their preferences or guided by case specifics instead of following the Endocrine Society or local guideline.

Currently, the ARR is the most reliable test for screening for PA [8, 9, 18, 19]. Confirmatory testing is recommended for all patients with a positive ARR to exclude the false-positive ARR results. However, this study shows the large variability in diagnostic work-up worldwide in which a confirmatory test was performed in only $32.9 \%$ of patients. The relatively low proportion of patients who underwent a confirmatory test and the large variability between the centers could be due to the fact that all confirmatory tests have some limitations and no universally accepted "gold standard" confirmatory test for PA is identified in the current literature [20-26]. Additionally, confirmatory tests are relatively expensive and frequently difficult to perform in outpatient settings $[8,9]$. This may have contributed to the changes in the 2016 guideline. Our data show that a relatively large proportion of patients $(40.7 \%)$ fulfills the triad of marked aldosterone, suppressed renin and hypokalemia. Omitting confirmatory testing in these patients would have been in agreement with the revised guideline of 2016. Therefore, this change in the guideline could induce a substantial reduction in confirmatory testing. It should be noted that this revised recommendation was based on a relatively low level of evidence, and therefore, not performing a confirmatory test is not without risks, especially because a patient with primary hypertension could be incorrectly diagnosed with PA and potentially undergo surgery based on the false-positive ARR results. Nevertheless, the recently published study by Umakasi et al. [27] supports the recommendation of omitting confirmatory testing in case of aldosterone excess $>20 \mathrm{ng} / \mathrm{dL}$ (550 pmol/L), suppressed renin and hypokalemia by presenting a PA diagnosis in $100 \%$ these cases.

There seems to be no consensus between clinicians on the use of AVS, as evidenced by the $34.7 \%$ of patients in this study who did not undergo AVS. Proponents argue that AVS should be considered as the "gold standard" for subtype testing, because multiple studies have shown its superiority over CT in determining disease lateralization. In these studies, the results of CT were compared to AVS as reference standard [11, 28, 29]. Opponents of AVS argue on the practical difficulties such as higher costs and the need of an interventional radiologist. This limits the wide availability of AVS, because some centers do not have the financial resources or expertise to perform AVS. In addition, AVS is an invasive procedure and also has failure and complication rates [10, 30, 31]. Furthermore, they argue that no significant differences in outcomes, such as antihypertensive medications or quality of life, were observed between CT and AVS within a randomized trial [32].

In this study, all participating medical centers used AVS in at least some patients. This suggests that AVS was available for all medical centers during some period of the inclusion period. Hence, we speculate that clinicians most likely chose to perform or not perform AVS based on their preferences or guided by case specifics. AVS was more frequently performed on patients with higher age, male gender, longer duration of hypertension and preoperative hypokalemia. Potentially, these represent the patients with more severe hypertension and/or hyperaldosteronism since some of these factors are also known as risk factors for less favorable clinical outcomes after adrenalectomy $[12,33,34]$. Furthermore, AVS was more frequently done in case of smaller tumor size, bilateral disease or normal adrenal anatomy on CT. Recently, Williams et al. also showed that AVS was more frequently performed in case of male gender and smaller tumor size on $\mathrm{CT}$ in univariable analysis. Furthermore, they indicated that AVS was used more often in case of lower blood pressure, higher ARR and lower estimated glomerular filtration rate [35].

The results of multivariable analysis within our cohort showed CT findings were independently associated with performing AVS. The presence of bilateral disease or normal adrenal anatomy on CT proved to be the most important trigger for clinicians to use AVS in daily practice. Likewise, $96.1 \%$ of patients with bilateral disease or normal adrenal anatomy on CT also underwent AVS. In contrast, patients with a clear unilateral nodule on CT and especially patients with larger tumors were less likely to undergo AVS. This further supports that CT findings most likely have the highest influence on the choice to perform or not perform AVS in daily clinical practice. Furthermore, hypokalemia proved to be independently associated with performing AVS. Nevertheless, it should be noted that Umakosi et al. [27] recently showed a higher percentage of hypokalemia in patients with APA compared to bilateral adrenal hyperplasia, $87 \%$ versus $21 \%$, respectively. Therefore, one could argue to perform AVS less frequently in case of hypokalemia. This seems to be in line with the 2016 guideline allowing the omission of AVS in case of a clear unilateral cortical adenoma on CT when this is combined with hypokalemia, age $<35$ years and marked aldosterone excess [9]. Within this study, only $6 \%$ of patients met these conditions and, consequently, this new 
recommendation only has marginal influence on daily practice.

Additionally, this study shows that work-up and surgery for PA also is performed in some centers with relatively low volume compared to centers in which PA is a clinical spearhead. This could have resulted in lower guideline adherence due to lower expertise and could stress the importance of further centralization of the treatment of PA. Furthermore, the low adherence can be due to the variety of diagnostic tests available of which some can be considered expensive, laborious or technically demanding. Also, it has been reported that it takes an average of 17 years for research evidence to reach clinical practice and, therefore, time to adoption of guidelines can take up to years or decades [36].

This study has some limitations. Similar to the majority of studies on PA, the retrospective design is a weakness. As a result, this study is more prone to missing data compared to prospective studies. Potentially, this could have led to lower rates of performed preoperative measurements of the ARR and confirmatory testing, as we chose to classify these modalities as not performed when the results were not known within the local patient files or referral letters. On the other hand, the retrospective design most likely is appropriate to evaluate different types of work-up strategies in clinical practice, as it reduces the influence of study protocols on decisions made by clinicians and therefore reflects daily practice. Because this is a surgical cohort, medically treated patients were not included. Therefore, we do not know if the results are representative for the medically treated PA population. Due to the different laboratory assays and reference values within the participating centers, we chose to not analyze absolute values of biochemical measurements. Therefore, marked aldosterone excess was defined as an aldosterone level above the local reference range, instead of the $>20 \mathrm{ng} / \mathrm{dL}(550 \mathrm{pmol} / \mathrm{L})$ cutoff suggested with the 2016 guideline [9]. Consequently, this could have influenced the $40.7 \%$ of patients meeting the criteria for omission of confirmatory testing.

The blood pressure-related outcomes within this cohort were published earlier and therefore not reported within this manuscript $[15,16]$. As presented within these and other recently published studies, complete cure of hypertension after the operation is far from a certainty $[12,15,16,37]$. Potentially, this is due to the large variability in work-up strategies which stresses the importance of evaluating how we currently perform the work-up to surgery for PA. Although this study showed no difference in cure of hypertension between patients with and without a work-up as recommended by the guidelines, our study cohort actually is not suitable for properly investigating the potential influence of the presented uniformity in work-up strategies on the outcomes after surgery. This is due to the retrospective design which is prone to confounding by indication.

In conclusion, this study examined the work-up to surgery for PA within current worldwide daily clinical practice. The results demonstrate large variability in work-up strategies both within and between the medical centers resulting in relatively low guideline adherence. If we want to further improve the benefits of surgery for PA in the future, we should strive for a more uniform work-up to surgery worldwide.

International CONNsortium study group* Rasa Zarnegar $\mathrm{MD}^{4}$, Thomas J. Fahey $\mathrm{MD}^{4}$, Frederick T. Drake MD ${ }^{5,6}$, Quan Y. Duh $\mathrm{MD}^{5}$, Stephanie D. Talutis MD ${ }^{6}$, David B. McAneny $\mathrm{MD}^{6}$, Catherine McManus $\mathrm{MD}^{7}$, James A. Lee $\mathrm{MD}^{7}$, Scott B. Grant MD ${ }^{8}$, Raymon H. Grogan MD ${ }^{9}$, Minerva A. Romero Arenas MD MPH ${ }^{10}$, Nancy D. Perrier $\mathrm{MD}^{10}$, Cord Sturgeon $\mathrm{MD}^{11}$, Tanya Castelino $\mathrm{MD}^{12}$, Elliot J. Mitmaker $\mathrm{MD}^{10}$, David N. Parente $\mathrm{MD}^{13}$, Jesse D. Pasternak $\mathrm{MD}^{13}$, Stan B. Sidhu MD ${ }^{14}$, Mark Sywak $\mathrm{MD}^{14}$, Gerardo D'Amato MD ${ }^{15}$, Marco Raffaelli $\mathrm{MD}^{16,17}$, Valerie Schuermans $\mathrm{MD}^{18}$, Nicole D. Bouvy $\mathrm{MD}^{18}$, Hasan H. Eker MD ${ }^{19}$, H. Jaap Bonjer MD ${ }^{19}$, Anton F. Engelsman $\mathrm{MD}^{19}$, Els J.M. Nieveen van Dijkum $\mathrm{MD}^{19}$, Michiel N. Kerstens $\mathrm{MD}^{20}$, Schelto Kruijff $\mathrm{MD}^{21}$. ${ }^{4}$ Department of Endocrine and Minimally Invasive Surgery, Weill Cornell Medical College, New York, USA. ${ }^{5}$ Department of Surgery, University of California San Francisco, San Francisco, USA. ${ }^{6}$ Department of Surgery, Boston University School of Medicine and Department of Graduate Medical Sciences, Boston, USA. ${ }^{7}$ Department of Endocrine Surgery, New York-Presbyterian-Columbia University, New York, USA. ${ }^{8}$ Department of Surgery, University of Chicago Medical Center, Chicago, USA. ${ }^{9}$ Department of Endocrine Surgery, Baylor St. Luke's Medical Center, Houston, USA. ${ }^{10}$ Department of Surgical Oncology, University of Texas MD Anderson Cancer Center, Houston, USA.

${ }^{11}$ Department of Surgery, Northwestern University Feinberg School of Medicine, Chicago, USA. ${ }^{12}$ Steinberg-Bernstein Centre for Minimally Invasive Surgery and Innovation, McGill University Health Centre, Montreal, Canada. ${ }^{13}$ Department of Surgery, University Health Network-Toronto General Hospital, Toronto, Canada. ${ }^{14}$ Department of Endocrine Surgery, Royal North Shore Hospital, Sydney, Australia. ${ }^{15}$ Department of Endocrine and Metabolic Surgery, Mater Olbia Hospital, Olbia, Italy. ${ }^{16}$ U.O.C. Chirurgia Endocrina e Metabolica, Fondazione Policlinico Universitario A. Gemelli IRCCS, Rome, Italy. ${ }^{17}$ Instituto di Semeiotica Chirurgica, Facolta di Medicina e Chirurgia, Universita Cattolica del Sacro Cuore, Rome, Italy. ${ }^{18}$ Department of Surgery, Maastricht University Medical Center+, Maastricht, the Netherlands. ${ }^{19}$ Department of Surgery, Amsterdam UMC, Amsterdam, the Netherlands. ${ }^{20}$ Department of Endocrinology, University Medical center Groningen, Groningen, the Netherlands. ${ }^{21}$ Department of Surgery, University Medical Center Groningen, Groningen, the Netherlands.

\section{Compliance with ethical standards}

Conflicts of interest The authors declare that there is no conflict of interest.

Open Access This article is licensed under a Creative Commons Attribution 4.0 International License, which permits use, sharing, adaptation, distribution and reproduction in any medium or format, as long as you give appropriate credit to the original author(s) and the source, provide a link to the Creative Commons licence, and indicate 
if changes were made. The images or other third party material in this article are included in the article's Creative Commons licence, unless indicated otherwise in a credit line to the material. If material is not included in the article's Creative Commons licence and your intended use is not permitted by statutory regulation or exceeds the permitted use, you will need to obtain permission directly from the copyright holder. To view a copy of this licence, visit http://creativecommons. org/licenses/by/4.0/.

\section{References}

1. Conn JW (1955) Presidential address. I. Painting background. II. Primary aldosteronism, a new clinical syndrome. J Lab Clin Med 45(1):3-17

2. Young WF (2007) Primary aldosteronism: renaissance of a syndrome. Clin Endocrinol (Oxf) 66(5):607-618

3. Douma S, Petidis K, Doumas M et al (2008) Prevalence of primary hyperaldosteronism in resistant hypertension: a retrospective observational study. Lancet 371(9628):1921-1926

4. Hannemann A, Wallaschofski H (2012) Prevalence of primary aldosteronism in patient's cohorts and in population-based studies-a review of the current literature. Horm Metab Res 44(3):157-162

5. Mills KT, Bundy JD, Kelly TN et al (2016) Global disparities of hypertension prevalence and control: a systematic analysis of population-based studies from 90 countries. Circulation 134(6):441-450

6. Käyser S (2016) Study heterogeneity and estimation of prevalence of primary aldosteronism: a systematic review and metaregression analysis. J Clin Endocrinol Metab 101(7):2826-2835

7. Piaditis G, Markou A, Papanastasiou L et al (2015) Progress in aldosteronism: a review of the prevalence of primary aldosteronism in pre-hypertension and hypertension. Eur $\mathrm{J}$ Endocrinol 172(5):191-203

8. Funder J, Carey R, Fardella C et al (2008) Case detection, diagnosis, and treatment of patients with primary aldosteronism: an Endocrine society clinical practice guideline. Eur J Endocrinol 93(9):3266-3281

9. Funder J, Carey R, Mantero F et al (2016) The management of primary aldosteronism: case detection, diagnosis, and treatment: an Endocrine society clinical practice guideline. J Clin Endocrinol Metab 101(5):1889-1916

10. Deinum J, Prejbisz A, Lenders JWM et al (2018) Adrenal vein sampling is the preferred method to select patients with primary aldosteronism for adrenalectomy: con side of the argument. Hypertension 71(1):10-14

11. Rossi GP, Funder JW (2018) Adrenal vein sampling is the preferred method to select patients with primary aldosteronism for adrenalectomy: pro side of the argument. Hypertension 71(1):5-9

12. Williams T, Lenders JWM, Mulatero P et al (2017) Outcomes after adrenalectomy for unilateral primary aldosteronism: an international consensus on outcome measures and analysis of remission rates in an international cohort. Lancet Diabetes Endocrinol 5(9):689-699

13. Zhou Y, Zhang M, Ke S et al (2017) Hypertension outcomes of adrenalectomy in patients with primary aldosteronism: a systematic review and meta-analysis. BMC Endocr Disord 17(1):61

14. Muth A, Ragnarsson O, Johannsson G et al (2015) Systematic review of surgery and outcomes in patients with primary aldosteronism. Br J Surg 102(4):307-317

15. Vorselaars WMCM, Nell S, Postma EL et al (2019) Clinical outcomes after unilateral adrenalectomy for primary aldosteronism. JAMA Surg. https://doi.org/10.1001/jamasurg.2018.5842
16. Vorselaars WMCM, van Beek D, Postma EL et al (2019) Clinical outcomes after surgery for primary aldosteronism: evaluation of the PASO-investigators' consensus criteria within a worldwide cohort of patients. Surgery. https://doi.org/10.1016/j.surg.2019. 01.031

17. van der Heijden Geert J M G, Donders ART et al (2006) Imputation of missing values is superior to complete case analysis and the missing-indicator method in multivariable diagnostic research: a clinical example. J Clin Epidemiol 59(10):1102-1109

18. Stowasser M, Gordon R, Gunasekera T et al (2003) High rate of detection of primary aldosteronism, including surgically treatable forms, after 'non-selective' screening of hypertensive patients. J Hypertens 21(11):2149-2157

19. Vorselaars WMCM, Valk GD, Vriens MR et al (2018) Case detection in primary aldosteronism: high-diagnostic value of the aldosterone-to-renin ratio when performed under standardized conditions. J Hypertens 36(7):1585-1591

20. Rossi GP, Belfiore A, Bernini G et al (2007) Prospective evaluation of the saline infusion test for excluding primary aldosteronism due to aldosterone-producing adenoma. J Hypertens 25(7):1433-1442

21. Mulatero P, Bertello C, Garrone C et al (2007) Captopril test can give misleading results in patients with suspect primary aldosteronism. Hypertension 50(2):26-37

22. Mulatero P, Milan A, Fallo F et al (2006) Comparison of confirmatory tests for the diagnosis of primary aldosteronism. J Clin Endocrinol Metab 91(7):2618-2623

23. Song Y, Yang S, He W et al (2018) Confirmatory tests for the diagnosis of primary aldosteronism: a prospective diagnostic accuracy study. Hypertension 71(1):118-124

24. Meng X, Li Y, Wang X et al (2018) Evaluation of the saline infusion test and the captopril challenge test in chinese patients with primary aldosteronism. J Clin Endocrinol Metab 103(3):853-860

25. Nanba K, Tamanaha T, Nakao K et al (2012) Confirmatory testing in primary aldosteronism. J Clin Endocrinol Metab 97(5):1688-1694

26. Willenberg HS, Vonend O, Schott M et al (2012) Comparison of the saline infusion test and the fludrocortisone suppression test for the diagnosis of primary aldosteronism. Horm Metab Res 44(7):527-532

27. Umakoshi H, Sakamoto R, Matsuda Y et al (2019) Role of aldosterone and potassium levels in sparing confirmatory tests in primary aldosteronism. J Clin Endocrinol Metab. https://doi.org/ 10.1210/clinem/dgz148

28. Lim V, Guo Q, Grant CS et al (2014) Accuracy of adrenal imaging and adrenal venous sampling in predicting surgical cure of primary aldosteronism. J Clin Endocrinol Metab 99(8):2712-2719

29. Kempers MJ, Lenders JW, van Outheusden L et al (2009) Systematic review: diagnostic procedures to differentiate unilateral from bilateral adrenal abnormality in primary aldosteronism. Ann Intern Med 151(5):329-337

30. Vonend O, Ockenfels N, Gao X et al (2011) Adrenal venous sampling: evaluation of the German Conn's registry. Hypertension 57(5):990-995

31. Harvey A, Kline G, Pasieka JL (2006) Adrenal venous sampling in primary hyperaldosteronism: comparison of radiographic with biochemical success and the clinical decision-making with "less than ideal" testing. Surgery 140(6):847-853

32. Dekkers T, Prejbisz A, Kool LJS et al (2016) Adrenal vein sampling versus CT scan to determine treatment in primary aldosteronism: an outcome-based randomised diagnostic trial. Lancet Diabetes Endocrinol 4:739-746

33. Burrello J, Burrello A, Stowasser M et al (2019) the primary aldosteronism surgical outcome score for the prediction of 
clinical outcomes after adrenalectomy for unilateral primary aldosteronism. Ann Surg. https://doi.org/10.1097/sla. 0000000000003200

34. Zarnegar R, Young W, Lee J et al (2008) The aldosteronoma resolution score: predicting complete resolution of hypertension after adrenalectomy for aldosteronoma. Ann Surg 247(3):511-518

35. Williams TA, Burrello J, Sechi LA, Fardella CE, Matrozova J, Adolf $\mathrm{C}$ et al (2018) Computed tomography and adrenal venous sampling in the diagnosis of unilateral primary aldosteronism. Hypertension 72(3):641-649
36. Moris ZS, Wooding S, Grant J (2011) The answer is 17 years, what is the question: understanding time lags in translational research. J R Soc Med 104(12):510-520

37. Vorselaars WMCM, van Beek D, Postma EL et al (2019) Validation of the aldosteronoma resolution score within current clinical practice. World J Surg 43(10):2459-2468. https://doi.org/ 10.1007/s00268-019-05074-z

Publisher's Note Springer Nature remains neutral with regard to jurisdictional claims in published maps and institutional affiliations. 